\title{
Measuring Fear of Failure
}

\section{Validation of a German Version of the Performance Failure Appraisal Inventory}

\author{
Christine Henschel@ and Benjamin Iffland® \\ Department of Psychology, Bielefeld University, Germany
}

\begin{abstract}
Fear of failure (FF) is a multidimensional construct encompassing anticipated negative consequences deriving from potential failures in evaluative achievement contexts, such as education or sports. The Performance Failure Appraisal Inventory (PFAl; Conroy et al., 2002) assesses five threat appraisals associated with FF and has been validated in various cultures, languages, and contexts. To date, there is no instrument measuring FF in an academic context in German. Thus, this study examined the psychometric properties of a German version of the PFAl in a sample of $N=326$ university students. Confirmatory factor analyses supported a correlated five-factor structure that has already been established in previous validation studies as well as a bifactor structure. All dimensions demonstrated satisfactory internal consistencies and construct validity. Within the scope of this study, the German version of the PFAl was found to be a psychometrically sound measure to assess FF in an academic context amongst university students.
\end{abstract}

Keywords: fear of failure, PFAl, questionnaire

Fear of failure $(\mathrm{FF})$ stems from the underlying fear of lacking the ability to achieve personally meaningful goals or to adequately demonstrate competence in achievement contexts, such as education or sports, where one's performance is evaluated against established standards (Atkinson, 1957; De Castella et al., 2013). It involves cognitive appraisals about the negative consequences of failing, the emotions that accompany these appraisals, and behavioral and motivational aspects aiming to reduce anticipated negative consequences through failure avoidance strategies (Sagar et al., 2011). This failure avoidance behavior can manifest in strategies such as selfhandicapping, performance-avoidance, or overstriving (Covington, 1992; Martin \& Marsh, 2003; McGregor \& Elliot, 2005). Individuals high in FF typically perceive failing to demonstrate sufficient competence or to perform adequately in evaluative achievement contexts as negative implication for their self-esteem and relational security (Conroy, 2001b; Conroy \& Elliot, 2004; Sagar et al., 2011).

FF has repeatedly been associated with the construct of achievement goal orientation (Conroy \& Elliot, 2004; Elliot \& Church, 1997). Achievement goal orientation refers to individuals' tendencies for approaching, engaging in, and evaluating their progress and performance in achievement contexts (Elliot \& Church, 1997). In their model, Elliot and Church (1997) distinguished between mastery-based goals focusing on the development of competence and performance-based goals focusing on the demonstration of competence. Competence has further been divided into task-based, self-based, and other-based competence, differentiating the frame of reference one's performance is evaluated in Elliot and McGregor (2001). In addition, competence was assumed to be valenced, and, therefore, approach and avoidance goals were subdivided (Elliot et al., 2011; Elliot \& McGregor, 2001). Thus, six goal constructs have been proposed: task-approach (TAP), taskavoidance (TAV), self-approach (SAP), self-avoidance (SAV), other-approach (OAP), and other-avoidance (OAV; Elliot et al., 2011; Wright et al., 2009). With these subdivisions, Elliot et al. (2011) assigned mastery-based goals to task-based and self-based goal orientations and performance-based goals to other-based goal orientations. For the association of FF and achievement goal orientation, positive correlations between FF and mastery- and performance-avoidance goals have been reported, as well as weaker but still significant correlations between FF and performance-approach goals (Conroy \& Elliot, 2004; Elliot \& Church, 1997; Elliot \& McGregor, 2001; Kahraman \& Sungur, 2016).

Overall, FF has been found across different levels of perceived and actual ability (Covington, 1992; Elliot \& Thrash, 2004). On the one hand, FF may serve as a motivating factor, in terms of striving for and reaching high levels of performance. However, it can also prevent individuals from actualizing their potential due to high anxiety and perceived incompetence (Conroy et al., 2002; 
Martin \& Marsh, 2003). Accordingly, individuals high in FF have reported generalizing a specific failure experience to the global self and, consequently, overgeneralize failure across various achievement contexts (McGregor \& Elliot, 2005). As a result, FF has been associated with different negative outcomes. In academic performance environments, FF went along with test- and trait-anxiety, depression, self-blame, low resilience, self-neglect, lower self-esteem, increased self-doubt, and a tendency to withdraw from academic pursuits (Conroy, 2001b; Covington, 1992; Elliot \& Church, 1997; Elliot \& McGregor, 2001; Martin \& Marsh, 2003). Moreover, FF has been associated with academic procrastination, decreased intrinsic motivation, and irrational cognitions (Haghbin et al., 2012).

\section{Measuring FF}

Early instruments measuring FF reflected unidimensional conceptualizations of $\mathrm{FF}$ and presented weak psychometrical properties (Gelbort \& Winer, 1985). Conroy (2001a) proposed a multidimensional instrument of $\mathrm{FF}$ to assess the strength of individuals' anticipation of different aversive consequences upon failure which was based on the conceptual framework of Birney et al. (1969) who argued that it is not the nonattainment of a goal itself that is feared by individuals high in FF, but rather the consequences that accompany failure. By analyzing interviews with athletes and performing artists, Conroy (2001a) identified 10 cognitive-motivational-relational appraisals associated with FF. Based on these, an initial 89-item Performance Failure Appraisal Inventory (PFAI) was developed. Exploratory factor analyses and calibration sample analyses reduced the PFAI to the final 25-item version (Conroy, 2001a; Conroy et al., 2002). The PFAI includes a set of five failure appraisals assessing the fear of (a) experiencing shame and embarrassment (FSE) regarding the belief that failure will bring shame and humiliation, (b) fear of devaluing one's self-estimate (FDS) regarding the belief that failure indicates the need to revise one's apparently overestimated self-appraisal, (c) fear of having an uncertain future (FUF) regarding the belief that failure upsets future plans, (d) fear of important others losing interest (FOL) regarding the belief that failure leads to negative relational consequences, such as decreasing social value, status, popularity and influence, and (e) fear of upsetting important others (FUO) regarding the belief that failure will disappoint significant others (Conroy et al., 2002).

The original PFAI has been found to be a reliable and valid instrument to assess $\mathrm{FF}$ in college students and athletes (Conroy et al., 2002; Conroy et al., 2003; Conroy \& Metzler, 2003; Sagar \& Jowett, 2010). The PFAI also demonstrated satisfactory internal consistency. In confirmatory factor analyses (CFA), both a correlated fivefactor model and a higher-order factor model revealed a satisfactory model fit (Conroy et al., 2002; Sagar \& Jowett, 2010). The model fit indicated that FF is a coherent construct that should be assessed based on its five different dimensions. Moreover, the majority of items showed a reasonable level of intraindividual stability (Conroy et al., 2003; Conroy \& Metzler, 2003). However, a reverse scored item of the FUF scale (Item 12: "When I am failing, I am not worried about it affecting my future plans") repeatedly demonstrated poor values. Therefore, it was recommended for revision by removing the word "not" (Conroy \& Metzler, 2003). For Item 16 of the FDS-scale ("When I am failing, I hate the fact that I am not in control of the outcome"), it was assumed that participants did not rate the belief, but rather the affective experience of "hate." Thus, Conroy and Metzler (2003) suggested reformulating this item into "When I am failing, my lack of control over the outcome bothers me."

Recently, validation studies for the PFAI were applied in different cultures, languages, and contexts. Portuguese (Correia et al., 2016), Turkish (Kahraman \& Sungur, 2016), and Romanian versions (Holic, 2018) have all demonstrated acceptable to satisfactory internal consistencies in athletes and students. Moreover, CFA replicated the correlated five-factor structure of the PFAI as it was presented for the original scale (Conroy et al., 2002). Construct validity of the Turkish version of the PFAI was examined through correlations between the PFAI subscales and the Achievement Goal Questionnaire (AGQ; Elliot \& McGregor, 2001). Kahraman and Sungur (2016) reported that all dimensions of the PFAI were positively correlated with mastery- and performance-avoidance goals and performance-approach goals, while no significant correlations between the PFAI subscales and masteryapproach goals were observed. In addition, Holic (2018) reported significant positive correlations between high levels of test anxiety and all PFAI subscales suggesting construct validity of the Romanian version of the PFAI. To date, a German version of the PFAI has not been published.

\section{Objectives and Aims}

The goal of the present study was to generate a German version of the PFAI and to evaluate its reliability and validity in an academic setting. As there is currently no measure assessing FF in German, an approximation of the construct validity of the PFAI was evaluated on basis of expected related constructs, namely achievement goal orientations, trait anxiety, and psychological symptoms. Based on previous findings (Conroy, 2001a; Conroy \& 
Elliot, 2004; Elliot \& Church, 1997; Elliot \& McGregor, 2001), overall FF as well as each of the subscales were assumed to be positively correlated with task-avoidance, self-avoidance, and OAV goal orientations, and to a lesser extent with OAP goal orientations. For task-avoidance, self-avoidance, and OAV goal orientations, we assumed medium-sized correlations, while $\mathrm{FF}$ and its subscales were expected to show small-sized correlations with OAP goal orientations. Regarding trait anxiety and psychological symptoms, overall $\mathrm{FF}$ and each of the subscales were additionally expected to be positively associated with trait anxiety, symptoms of depression, social anxiety symptoms, and symptoms of distrust, as FF has been associated with different negative psychological outcomes, such as depression, anxiety, and higher levels of interpersonal distress (Conroy, 2001b; Conroy et al., 2009; Elliot \& McGregor, 2001; Wright et al., 2009). Here, we expect to find small- to medium-sized associations of overall $\mathrm{FF}$ and the subscales, reflecting that although $\mathrm{FF}$ is related to psychopathology and trait anxiety, it is still a distinct construct. To recruit participants and assess data, a webbased Internet survey was used. It was expected that this recruitment would allow for the study of a large sample of participants from diverse academic backgrounds presenting with a wide range of $\mathrm{FF}$.

\section{Method}

\section{Participants}

The survey was advertised through social media networks and the universities' research platforms. In order to be included in the present study, participants had to be between the ages of 18 and 65, hold an active university student status, and possess sufficient knowledge of the German language (clearly able to understand the information and instructions). Initially, $N=352$ university students participated in the present study, and $n=26$ participants were excluded due to incomplete or suspicious data, for example, regarding the amount of time needed for completing the questionnaire. No further exclusion criteria were applied. The final sample consisted of $N=326$ university students of whom $n=251$ (77.0\%) were female. Participants' ages ranged from 18 to 54 years $(M=24.19 ; S D=4.91)$. Participants' nationality $(n=306$; 93.9\%) and native language $(n=301 ; 92.3 \%)$ were predominantly German, with smaller percentages of other nationalities $(n=20,6.11 \%)$ and native languages $(n=25$, $7.7 \%)$. Overall, participants reported diverse academic backgrounds regarding study course, year of study, degrees, and universities. Each participant read and signed an informed consent form that was approved by the Ethics Committee of Bielefeld University (protocol number: EUB 2020-027).

\section{Instruments}

The 25 items of the original PFAI (Conroy et al., 2002) were translated and adapted into German by one of the authors. Following the recommendation of Conroy et al. (2003), the translation of Item 12 of the FUF-subscale was based on the adapted item by removing the word not: "When I am failing, I worry about it affecting my future plans." The items were then back-translated into English by an independent researcher and reviewed for coherence with the initial items by one of the original PFAI authors, David E. Conroy. The final German version of the PFAI used in the present study (see Electronic Supplementary Material 1 [ESM 1]) consisted of 25 items measuring five appraisals associated with anticipated negative consequences upon failure in an academic context: fear of experiencing shame and embarrassment (FSE; 7 items), FDS (4 items), FUF (4 items), FOL (5 items), and FUO (5 items). Participants were asked to rate the extent to which the items' statements applied to their studies on a 5-point rating scale $(1=$ does not apply at all to $5=$ completely true), with higher scores indicating greater levels of FF.

To assess achievement goals for their academic performance, participants completed the AGQ (Elliot et al., 2011). The measure contained 18 items to assess six goal constructs with three items each: TAP, TAV, SAP, SAV, $\mathrm{OAP}$, and OAV. In the present study, AGQ-scales demonstrated satisfactory to excellent internal consistencies ranging from $\alpha=.67$ (TAV) to $\alpha=.94(\mathrm{OAP})$. As the AGQ has previously been applied in a German-speaking sample (e.g., Elliot et al., 2011; Harrer, 2012), the German version was adopted from Harrer (2012) who obtained the German AGQ "directly from the authors of the model" (p. 38). The instructions were adapted for this study, asking the participants to indicate the extent to which the stated achievement goals applied to the goals they had or did not have for exams in their studies on a 7-point rating scale $(1=$ does not apply at all to $7=$ completely true $)$.

The Trait-Anxiety-scale of the German version of the State-Trait Anxiety Inventory (STAI-T; Laux et al., 1981) was used to measure anxiety level as a personal characteristic. The scale contained 20 items, rated on a 4-point rating scale $(1=$ almost never to $4=$ almost always $)$. In the present study, the STAI-T demonstrated an excellent internal consistency $(\alpha=.93)$. Higher scores indicated higher levels of anxiety.

The Symptom-Checklist-27 (SCL-27; Hardt et al., 2004) was used as a short multidimensional screening instrument 
for mental health problems. It contained 27 items on six scales regarding current psychological symptoms: symptoms of depression, dysthymic symptoms, vegetative symptoms, agoraphobic symptoms, social anxiety symptoms, and symptoms of distrust. Items were rated on a 5-point rating scale $(1=$ not at all to $5=$ extremely). Internal consistencies for the different scales demonstrated satisfactory values in the present study, ranging from $\alpha=.70$ (agoraphobic symptoms and symptoms of distrust) to $\alpha=.81$ (dysthymic symptoms).

\section{Procedure}

The study web page was implemented using Qualtrics software (Qualtrics, Provo, UT). Data were collected over a 3 -month period. On entering the web page, participants were informed about the content and purpose of the study as well as how their submitted data will be used and saved. They were further informed that their participation was voluntary, data were collected anonymously, and that they could withdraw their participation in the study at any time, initiating a deletion of all their data submitted up to that point. After giving their written informed consent, participants were asked to fill out a sociodemographic questionnaire, followed by the above-mentioned study questionnaires. On the last page, participants were given the chance to contact the researcher via e-mail in case of further questions about the study or to request a deletion of their submitted data.

\section{Statistical Analyses}

All statistical analyses were carried out using RStudio Version 3.4.3 (RStudio Team, 2020) and SPSS Version 21.0 (IBM Corporation, 2012). To indicate the internal consistency, Cronbach's alpha $(\alpha)$, McDonald's omega total $(\omega t)$, and omega hierarchical $(\omega h)$ were estimated for the overall PFAI and all subscales. Additionally, intercorrelations between the PFAI subscales were calculated to indicate the extent to which the scales overlap. Associations of the total PFAI sore and all subscales with sociodemographic variables were examined using Pearson correlations and multivariate ANOVAs. When indicated, additional post-hoc univariate ANOVAs and $t$-tests were conducted. CFA were conducted for five different models to identify the model structure that best captured the dimensions of the PFAI. Corresponding with the models initially tested by Conroy et al. (2002) and Sagar and Jowett (2010), four models tested in this study were as follows: (M1) a single factor representing FF, (M2) five uncorrelated factors representing the specific $\mathrm{FF}$ appraisals, (M3) five correlated factors representing the specific FF appraisals, and (M4) a higher-order factor that accounts for the correlations amongst the five first-order factors. The fifth model tested (M5) was a bifactor model to examine whether the five factors are contributing unique information to $\mathrm{FF}$, by each having their own direct loading on FF. This model was added to the list of previously tested models since past research indicated that the fit of bifactor models is often superior to higher-order models in CFA (Cucina \& Byle, 2017). Within this framework, reported fit indices included the $\chi^{2}$ test, the RMSEA, the standardized root mean square residual (SRMR), the comparative fit index (CFI), the non-normed fit index (NNFI), the goodness of fit index (GFI), and the Akaike information criterion (AIC). In the present study, values of $\geq .95$ were adopted to indicate an acceptable fit for the GFI, NNFI, and CFI, while eliminating the risk of overfitting (Hooper et al., 2008; Hu \& Bentler, 1999). For the RMSEA and SRMR, values of $<.08$ were considered satisfactory (Hooper et al., 2008; Hu \& Bentler, 1999). Regarding the AIC, there is no specified acceptable value but when comparing different models, the one with the lowest AIC score is suggested as the most parsimonious (Wagenmakers \& Farrell, 2004). For each model tested in the CFA, omega values were assessed according to the guidelines for choosing the appropriate omega estimates provided by Flora (2020).

Construct validity was tested using Pearson correlations examining the association between PFAI scores and scales measuring achievement goal orientation, trait anxiety, and psychopathology. Regarding the interpretation of Pearson correlations, Cohen (1988) considered values of $r=.10$ to represent a small-sized, $r=.30$ a medium-sized, and $r=.50$ a large-sized effect. Moreover, regression analyses were conducted to examine unique associations of the PFAI subscales with achievement goal orientation, trait anxiety, and psychopathology. Finally, a hierarchical multiple regression analysis was applied to assess whether the PFAI subscales significantly improved the ability to predict trait anxiety. Here, psychopathology as measured by the SCL-27 subscales and the PFAI subscales were used as predictors.

\section{Results}

\section{Descriptive Data}

The overall PFAI and its subscales demonstrated satisfactory to excellent levels of internal consistencies ranging from $\alpha=.77$ for FDS to $\alpha=.93$ for overall FF, from $\omega t=.80$ for FDS to $\omega t=.94$ for overall $\mathrm{FF}$, and from $\omega h=.72$ for overall $\mathrm{FF}$ to $\omega h=.89$ for FUF (Table 1). Intercorrelations 
Table 1. Internal consistencies and intercorrelations of the PFAI

\begin{tabular}{llllllll}
\hline & FSE & FDS & FUF & FOL & $\alpha$ & $\omega t$ & $\omega h$ \\
\hline PFAl & & & & & .93 & .94 & .72 \\
FSE & 1 & & & & .88 & .91 & .82 \\
FDS & $.59^{* *}$ & 1 & & & .77 & .80 & .77 \\
FUF & $.53^{\star *}$ & $.53^{\star *}$ & 1 & & .90 & .91 & .89 \\
FOL & $.62^{\star \star}$ & $.33^{\star *}$ & $.33^{\star *}$ & 1 & .86 & .89 & .83 \\
FUO & $.45^{\star *}$ & $.21^{\star *}$ & $.34^{\star *}$ & $.57^{\star *}$ & .82 & .84 & .75 \\
\hline
\end{tabular}

Note. FDS = fear of devaluing one's self-estimate; FSE = fear of experiencing shame and embarrassment; FUF = fear of having an uncertain future; FUO = fear of upsetting important others; PFAI = Performance Failure Appraisal Inventory; $\alpha=$ Cronbach's alpha; $\omega t=$ McDonald's omega total; $\omega h=$ McDonald's omega hierarchical. ${ }^{*} p<.05,{ }^{*} p<.01$.

between subscales were moderate. Correlations between the single items were also moderate (see ESM 2). Table 2 provides information on items' $M, S D$, factor loadings, and error variances of the correlated five-factor model (M3), the higher-order factor model (M4), and the bifactor model (M5). All item mean scores were low to moderate relative to the response scale of 1-5, ranging from $M=1.52$ $(S D=0.89)$ for Item 17 to $M=3.73(S D=1.11)$ for Item 16 . Overall, factor loadings were moderate to high, ranging from .47 for Item 16 to .89 for Item 8 . The lowest factor loading and the highest error variance were observed for Item 16. Standardized factor loadings based upon correlation matrix are presented in Table 3.

Analyses of associations of the total PFAI score and the PFAI subscales with sociodemographic variables revealed that the German version of the PFAI was not significantly correlated with age (all $p s>.05 / 6$ ). On a multivariate level, PFAI scores differed significantly between gender (Wilks $\lambda=.95, F[5,317]=3.34, p=.006$, partial $\eta^{2}=0.050$ ). However, post-hoc analyses did not show significant gender differences on the total scale and subscales (all ps $>.05 / 6$ ). Furthermore, no significant differences were found with regards to citizenship, native language, and family status (all multivariate $p s>.05$ ), though scores differed significantly between study courses on a multivariate level (Wilks $\lambda=.76, F[60,1,427.291]=1.44$, $p=.016$, partial $\left.\eta^{2}=0.054\right)$. While post-hoc analyses confirmed a significant difference between courses for the FOL-subscale $(F[12,308]=2.58, p=.003$, partial $\eta^{2}=0.091$ ), all other post-hoc ANOVAs did not show significance (all $p s>.05 / 6)$.

\section{Confirmatory Factor Analyses}

Regarding the CFA (Table 4), values of the fit indices as well as the AIC indicate the superiority of the bifactor model (M5), followed closely by the correlated five-factor model (M3). For both models, the RMSEA and SRMR just
Table 2. $M, S D$, factor loadings, and error variances of the correlated five-factor model (M3), the higher-order model (M4), and the bifactor model (M5)

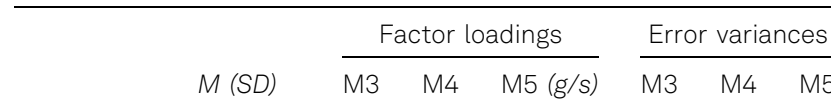

Fear of experiencing shame and embarrassment

$\begin{array}{llllllll}\text { Item 10 } & 2.58(1.34) & .67 & .67 & .74 /-.01 & .55 & .56 & .46 \\ \text { Item 15 } & 3.61(1.26) & .70 & .69 & .73 / .09 & .51 & .52 & .46 \\ \text { Item 18 } & 3.52(1.25) & .74 & .75 & .60 / .53 & .45 & .44 & .35 \\ \text { Item 20 } & 2.63(1.31) & .73 & .73 & .66 / .28 & .48 & .47 & .48 \\ \text { Item 22 } & 3.06(1.32) & .70 & .70 & .63 / .26 & .51 & .50 & .53 \\ \text { Item 24 } & 3.41(1.35) & .81 & .81 & .68 / .54 & .34 & .34 & .25 \\ \text { Item 25 } & 3.21(1.39) & .68 & .68 & .59 / .34 & .54 & .54 & .54\end{array}$

Fear of devaluing one's self-estimate

$\begin{array}{llllllll}\text { Item 1 } & 2.11(1.03) & .60 & .61 & .43 / .43 & .65 & .63 & .63 \\ \text { Item } 4 & 2.63(1.21) & .82 & .84 & .56 / .67 & .32 & .30 & .24 \\ \text { Item 7 } & 2.96(1.30) & .86 & .84 & .61 / .56 & .27 & .29 & .32 \\ \text { Item 16 } & 3.73(1.11) & .47 & .47 & .50 / .12 & .78 & .78 & .74\end{array}$

Fear of having an uncertain future

$\begin{array}{llllllll}\text { Item 2 } & 3.14(1.22) & .71 & .71 & .59 / .42 & .49 & .50 & .47 \\ \text { Item 5 } & 3.29(1.16) & .87 & .87 & .57 / .66 & .24 & .24 & .24 \\ \text { Item 8 } & 3.23(1.19) & .89 & .89 & .49 / .77 & .20 & .20 & .16 \\ \text { Item 12 } & 3.55(1.18) & .87 & .87 & .54 / .67 & .25 & .25 & .26\end{array}$

Fear of important others losing interest

$\begin{array}{llllllll}\text { Item 11 } & 2.44(1.25) & .81 & .82 & .70 / .44 & .35 & .32 & .31 \\ \text { Item 13 } & 1.79(1.01) & .64 & .63 & .42 / .47 & .59 & .61 & .60 \\ \text { Item 17 } & 1.52(0.89) & .68 & .66 & .42 / .53 & .54 & .57 & .54 \\ \text { Item 21 } & 2.06(1.17) & .82 & .83 & .51 / .70 & .32 & .32 & .25 \\ \text { Item 23 } & 2.26(1.23) & .78 & .78 & .59 / .51 & .39 & .39 & .39\end{array}$

Fear of upsetting important others

\begin{tabular}{llllllll} 
Item 3 & $1.81(1.07)$ & .68 & .70 & $.26 / .69$ & .54 & .51 & .46 \\
Item 6 & $2.33(1.29)$ & .64 & .67 & $.44 / .50$ & .59 & .56 & .55 \\
Item 9 & $1.54(0.89)$ & .67 & .65 & $.41 / .50$ & .56 & .58 & .59 \\
Item 14 & $2.04(1.11)$ & .64 & .62 & $.35 / .51$ & .59 & .61 & .62 \\
Item 19 & $1.94(1.11)$ & .85 & .85 & $.43 / .73$ & .28 & .27 & .28 \\
\hline
\end{tabular}

Note. $\mathrm{g} / \mathrm{s}=$ general factor/scale; $\mathrm{M} 3=$ correlated five-factor model; M4 = higher-order model; M5 = bifactor model.

met the proposed criteria of $<.08$ for a satisfactory fit, while the CFI, GFI, and NNFI failed to meet the proposed criteria of $\geq .95$ for an acceptable fit. Factor correlations of the correlated five-factor model (M3) and coefficient omega estimates for the models tested in the CFA are presented in Table 5 and Table 6.

\section{Construct Validity}

Regarding construct validity (Table 7), overall FF, and FSE showed significant positive small-sized correlations with 
Table 3. Standardized factor loadings based upon correlation matrix with five extracted factors

\begin{tabular}{|c|c|c|c|c|c|}
\hline & Factor 1 & Factor 2 & Factor 3 & Factor 4 & Factor 5 \\
\hline \multicolumn{6}{|c|}{ Fear of experiencing shame and embarrassment } \\
\hline Item 10 & .20 & .35 & & .41 & \\
\hline Item 15 & .43 & .32 & & & \\
\hline Item 18 & .77 & & & & \\
\hline Item 20 & .49 & & & & \\
\hline Item 22 & .50 & & & .21 & \\
\hline Item 24 & .80 & & & & \\
\hline Item 25 & .55 & & & & \\
\hline \multicolumn{6}{|c|}{ Fear of devaluing one's self-estimate } \\
\hline Item 1 & & .65 & & & \\
\hline Item 4 & & .81 & & & \\
\hline Item 7 & & .78 & & & \\
\hline Item 16 & .25 & .24 & & & \\
\hline \multicolumn{6}{|c|}{ Fear of having an uncertain future } \\
\hline Item 2 & & .27 & .54 & & \\
\hline Item 5 & & & .83 & & \\
\hline Item 8 & & & .92 & & \\
\hline Item 12 & & & .90 & & \\
\hline \multicolumn{6}{|c|}{ Fear of important others losing interest } \\
\hline Item 11 & & & & .68 & \\
\hline Item 13 & & & & .61 & \\
\hline Item 17 & & & & .71 & .21 \\
\hline Item 21 & & & & .78 & \\
\hline Item 23 & & & & .69 & \\
\hline \multicolumn{6}{|c|}{ Fear of upsetting important others } \\
\hline Item 3 & & & & & .77 \\
\hline Item 6 & & & & & .64 \\
\hline Item 9 & & & & .48 & .39 \\
\hline Item 14 & & & & .21 & .44 \\
\hline Item 19 & & & & .20 & .69 \\
\hline
\end{tabular}

SAP, SAV, OAP, and OAV goals. FDS also showed significant positive small-sized correlations with SAV and OAV goals. FUF, important others losing interest and upsetting important others further showed significant positive small-sized correlations with SAP goals. FUF showed significant positive small-sized correlations with SAV goals. Further, FUO showed significant negative smallsized correlations with TAP and TAV goals.

Overall FF, FSE and FDS showed significant positive large-sized correlations with trait anxiety, while all other subscales showed significant positive medium-sized correlations with trait anxiety. Regarding current psychological symptoms, overall FF and all subscales showed significant positive correlations with all symptoms assessed.

Regression analyses examining unique contributions of the PFAI subscales to predict achievement goal orientation, trait anxiety, and psychopathology are presented in Table 8. PFAI subscales show different patterns of specific associations with external criteria. Moreover, the PFAI subscales made a significant incremental contribution of variance $(6 \%)$ to the prediction of trait anxiety beyond the variance explained by psychopathology (see Table 9). However, only the subscale FSE was a significant predictor of trait anxiety.

\section{Discussion}

The present study examined the psychometric properties of a German version of the PFAI. In order to determine whether this measure can be used to assess FF in an academic context among German speaking student populations, the extent to which students fear different potential negative consequences upon failure in their academic studies was evaluated. Internal consistencies for the overall PFAI and all subscales proved to be satisfactory to excellent. In line with previous studies, the internal consistencies for FSE and FOL appeared to be among the highest (Conroy et al., 2002; Correia et al., 2016; Holic, 2018; Kahraman \& Sungur, 2016; Sagar \& Jowett, 2010), and FDS demonstrated the lowest internal consistencies among subscales (Conroy et al., 2002; Correia et al., 2016; Kahraman \& Sungur, 2016; Sagar \& Jowett, 2010). However, the FUF scale demonstrated high internal

Table 4. Fit indices of the CFA

\begin{tabular}{lcccccccc}
\hline & $d f$ & $\chi^{2}$ & RMSEA [90\% Cl] & SRMR & CFI & GFI & NNFI \\
\hline M1 & 275 & $2,150.30$ & $.15[.14-.15]$ & .12 & .58 & .56 & .54 & $22,950.50$ \\
M2 & 275 & $1,287.45$ & $.11[.10-.11]$ & .27 & .77 & .75 & .75 & $22,087.65$ \\
M3 & 265 & 753.43 & $.08[.07-.08]$ & .07 & .89 & .84 & .88 & $21,573.63$ \\
M4 & 270 & 841.72 & $.08[.08-.09]$ & .09 & .87 & .83 & .86 & $21,651.92$ \\
M5 & 250 & 665.96 & $.07[.07-.08]$ & .08 & .91 & .86 & .89 & $21,516.16$ \\
\hline
\end{tabular}

Note. $\mathrm{AIC}=$ Akaike Information Criterion; $\mathrm{CFA}=$ confirmatory factor analyses; $\mathrm{CFI}=$ comparative fit index; $d f=$ degrees of freedom; GFI = goodness of fit index; $\mathrm{NNFI}=$ non-normed fit index; SRMR = standardized root mean square residual; $90 \% \mathrm{Cl}=90 \% \mathrm{Cl}$ of RMSEA; $\mathrm{M} 1=$ one-factor model; $\mathrm{M} 2=$ uncorrelated fivefactor model; M3 = correlated five-factor model; M4 = higher-order model; M5 = bifactor model. 
Table 5. Factor correlations of the correlated five-factor model (M3)

\begin{tabular}{lcclll}
\hline & FSE & FDS & FUF & FOL & FUO \\
\hline FSE & 1 & & & & \\
FDS & .66 & 1 & & & \\
FUF & .56 & .56 & 1 & & \\
FOL & .69 & .37 & .36 & 1 & \\
FUO & .50 & .18 & .37 & .67 & 1 \\
\hline
\end{tabular}

Note. FDS = fear of devaluing one's self-estimate; FOL = fear of important others losing interest; FSE = fear of experiencing shame and embarrassment; FUF = fear of having an uncertain future; FUO = fear of upsetting important others.

consistencies in the present study, which is in contrast to previous results (Holic, 2018; Kahraman \& Sungur, 2016; Sagar \& Jowett, 2010). Subscale-intercorrelations were moderate and also comparable to previous studies (Sagar \& Jowett, 2010; Sagar \& Stoeber, 2009). Findings in the present study further supported the correlated five-factor structure established in previous validation studies and demonstrated an adequate approximation of construct validity for the German version of the PFAI. However, the present study revealed that the data structure of the German version was slightly better reflected in a bifactor model.

In the present study, the bifactor model and the correlated five-factor model generated the best fit to data in comparison to other models. Although fit indices in previous studies demonstrated a better model fit overall (see Table 10), results from the present study are basically in line with previous findings in other versions of the PFAI, which also identified the correlated five-factor model to have the best model fit (Conroy et al., 2002; Correia et al., 2016; Holic, 2018; Kahraman \& Sungur, 2016; Sagar \& Jowett, 2010). The previously established fit of the correlated five-factor model leads to the suggestion that $\mathrm{FF}$ by itself does not represent a coherent construct and should thus be assessed using its different dimensions. Accordingly, the superior bifactor model suggests that the five factors are contributing unique information to FF. On the other hand, however, each subscale has its own direct

Table 6. Coefficient omega estimates for the models tested in the CFA

\begin{tabular}{lcccccc}
\hline & FSE & FDS & FUF & FOL & FUO & General \\
\hline M1 & & & & & & .93 \\
M2 & .88 & .80 & .90 & .87 & .82 & \\
M3 & .88 & .81 & .90 & .87 & .82 & \\
M4 & .88 & .81 & .90 & .87 & .83 & .82 \\
M5 & .14 & .34 & .52 & .43 & .58 & .83 \\
\hline
\end{tabular}

Note. CFA = confirmatory factor analyses; FDS = fear of devaluing one's selfestimate; FSE = fear of experiencing shame and embarrassment; FOL = fear of important others losing interest; FUF = fear of having an uncertain future; FUO = fear of upsetting important others; M1 = one-factor model;

M2 = uncorrelated five-factor model; M3 = correlated five-factor model; M4 = higher-order model; M5 = bifactor model. loading on the general factor of $\mathrm{FF}$ emphasizing on the significance of the total PFAI score. Also, when looking at omega estimates for the bifactor model, Flora (2020) stated that low omega estimates for the specific factors and a high omega estimate for the general factor suggests that the reliable variances of the subscales are attributable to the general factor, in this case $\mathrm{FF}$, instead of representing independent aspects of FF. Hence, the correlated five-factor model and the bifactor model suggest the use of the total PFAI score and its subscales when drawing conclusions from test scores. On the one hand, the total PFAI score could be insufficient for the interpretation of $\mathrm{FF}$, as data suggest that scoring high in one dimension does not necessarily mean that the total FF score is high. On the other hand, using the total PFAI score improves validity, reliability, and consistency of the test, since the subscales are not fully independent constructs of FF. This should be considered when establishing cut-off scores for the PFAI and its subscales.

A reason for the slightly inferior model fit in the present study, compared to previous validation studies, may be that the data were collected across various study years, degrees, courses, ethnical backgrounds, ages and universities across Germany, generating a rather heterogeneous sample. Accordingly, Muthén (1989) showed that population homogeneity affects model fit and model

Table 7. Construct validity (Pearson's $r$ ) for the PFAl subscales

\begin{tabular}{|c|c|c|c|c|c|c|}
\hline & FSE & FDS & FUF & FOL & FUO & PFAI \\
\hline$A G Q$ - TAP & -.02 & -.05 & .02 & -.06 & $-.14^{\star}$ & -.06 \\
\hline$A G Q$ - TAV & -.02 & -.03 & .04 & -.08 & $-.11^{\star}$ & -.05 \\
\hline$A G Q$ - SAP & $.16 * \star$ & .09 & $.12^{\star}$ & $.14 * \star$ & $.12^{\star}$ & $.17 \star \star$ \\
\hline$A G Q$ - SAV & $.16 \star \star$ & $.20 * \star$ & $.14^{\star}$ & .02 & -.02 & $.13^{\star}$ \\
\hline$A G Q$ - OAP & $.19 * \star$ & .09 & .04 & .09 & .09 & $.15^{\star \star}$ \\
\hline$A G Q$ - OAV & $.26 * \star$ & $.22 * \star$ & .09 & .09 & .07 & $.21 \star \star$ \\
\hline STAI-T & $.59 \star \star$ & $.50 \star \star$ & $.45^{\star \star}$ & $.40 \star \star$ & $.38 \star \star$ & $.63^{\star \star}$ \\
\hline SCL-27 - DEP & $.40 * \star$ & $.38 * \star$ & $.36 * \star$ & $.35^{\star \star}$ & $.35 \star \star$ & $.48^{\star \star}$ \\
\hline SCL-27 - DYS & $.32 \star \star$ & $.30 * \star$ & $.23^{\star \star}$ & $.23 * \star$ & $.22^{\star}$ & $.35^{\star \star}$ \\
\hline SCL-27 - VEG & $.24 * \star$ & $.20 * *$ & $.22^{\star \star}$ & $.13^{*}$ & $.16 \star \star$ & $.26 * \star$ \\
\hline$S C L-27-A G O$ & $.33^{\star \star}$ & $.23^{\star \star}$ & $.30 \star \star$ & $.18 * \star$ & $.20 * \star$ & $.34^{\star \star}$ \\
\hline SCL-27 - SOC & $.53 \star \star$ & $.46^{\star \star}$ & $.37 * \star$ & $.31 \star \star$ & $.29 * \star$ & $.53 \star \star$ \\
\hline SCL-27 - MIS & $.35 * \star$ & $.29 * \star$ & $.26 * \star$ & $.43^{\star *}$ & $.35 * *$ & $.44^{\star}$ \\
\hline
\end{tabular}

Note. $A G O=$ agoraphobic symptoms; $A G Q=$ Achievement Goal Questionnaire; DEP = symptoms of depression; DYS = dysthymic symptoms; FDS = fear of devaluing one's self-estimate; FOL = fear of important others losing interest; FSE = fear of experiencing shame and embarrassment; FUF = fear of having an uncertain future; FUO = fear of upsetting important others; MIS = symptoms of mistrust; OAP = other-approach; OAV = other avoidance; PFAI = Performance Failure Appraisal Inventory; SAP = selfapproach; SAV = self-avoidance; SCL-27 = The Symptom-Checklist-27; SOC = sociophobic symptoms; STAI-T = trait-anxiety-scale of the State-Trait Anxiety Inventory; TAP = task-approach; TAV = task-avoidance;

VEG $=$ vegetative symptoms. ${ }^{\star} p<.05,{ }^{* \star} p<.01$. 
Table 8. Unique associations of the PFAl subscales with external criteria

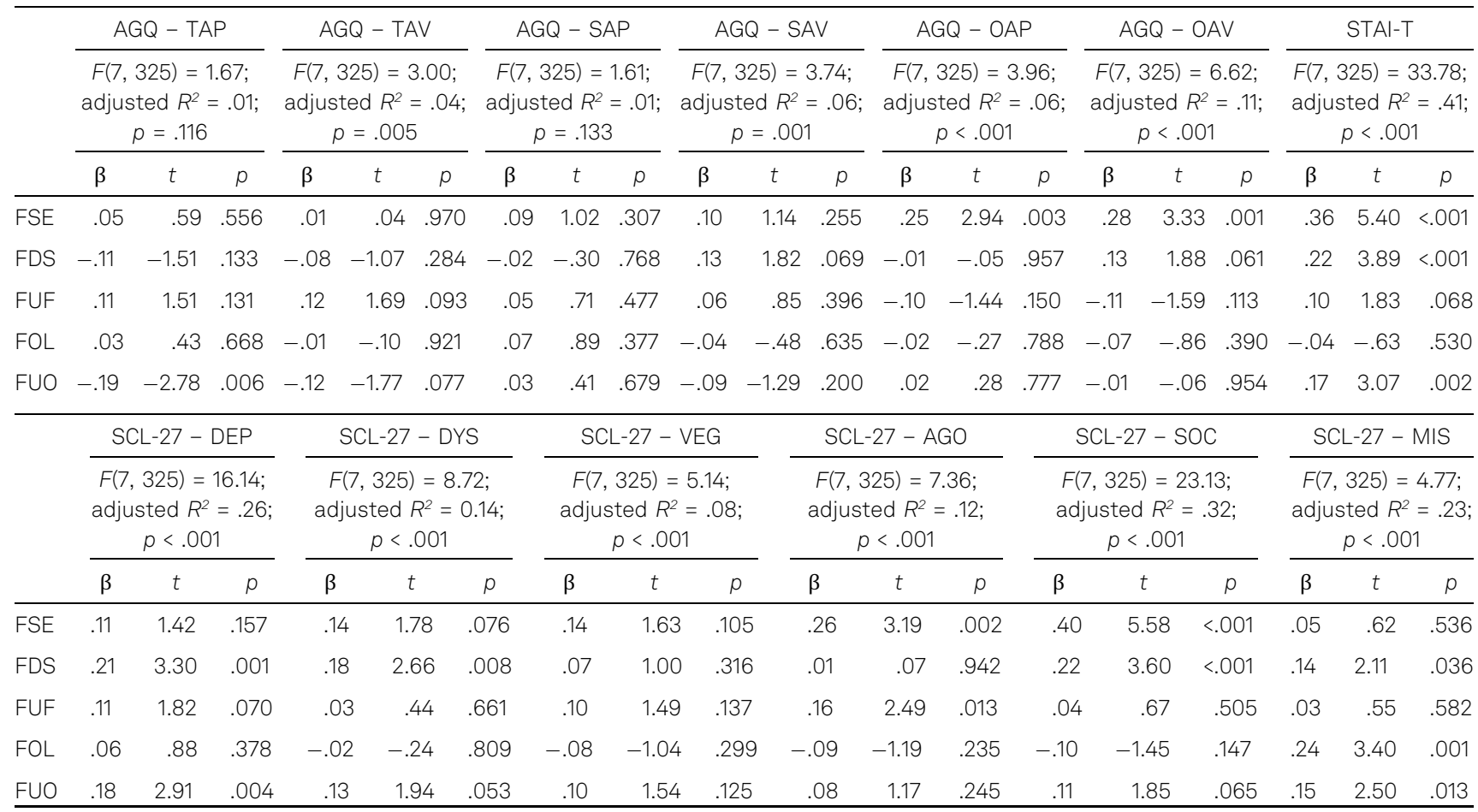

Note. All multiple linear regression models were adjusted for age and gender. AGO = agoraphobic symptoms; AGQ = Achievement Goal Questionnaire; DEP = symptoms of depression; DYS = dysthymic symptoms; FDS = fear of devaluing one's self-estimate; FOL = fear of important others losing interest; FSE = fear of experiencing shame and embarrassment; FUF = fear of having an uncertain future; FUO = fear of upsetting important others; MIS = symptoms of mistrust; OAP = other-approach; OAV = other-avoidance; SAP = self-approach; SAV = self-avoidance; SCL-27 = The Symptom-Checklist-27; SOC = sociophobic symptoms; STAI-T = trait-anxiety-scale of the State-Trait Anxiety Inventory; TAP = task-approach; TAV = task-avoidance; VEG = vegetative symptoms.

rejection in CFA. Accordingly, samples of previous validation studies were more homogeneous, exclusively consisting of school-children (Holic, 2018; Kahraman \& Sungur, 2016) or athletes and sport club members (Correia et al., 2016; Sagar \& Jowett, 2010). This assumption is also supported by present analyses of associations of the total PFAI score and the PFAI subscales with sociodemographic variables revealing multivariate differences related to gender and study courses. However, we were not able to assess measurement invariance and differences in model fit between subgroups in our sample because it was not balanced with respect to gender or study courses. Therefore, conclusions regarding the influence of sample characteristics on model fit have to be drawn with caution.

Nevertheless, the results of the CFA indicate that either the bifactor model structure or the correlated five-factor structure is most appropriate for the German version of the PFAI among student populations with various academic backgrounds, though further investigation to support this conclusion is needed. Additionally, except for one adapted item (Item 12), no items had to be modified or deleted during the course of this study to create a better model fit as was necessary in the Portuguese validation study (Correia et al., 2016).
Although model fit indexes were similar to previous validation studies, the German version failed to meet cutoffs for acceptable model fit with respect to the CFI, GFI, and NNFI (Hooper et al., 2008; Hu \& Bentler, 1999). Although the use of cut-off scores has been recently questioned (Greiff \& Heene, 2017; Moshagen \& Auerswald, 2018), local misfit inspection was applied. Based on a critical review of factor loadings and residual covariances in models M3 and M5 (Heene et al., 2011), it may be suggested that weakness of fit was due to Items 10, 15, 18, and 22 of the FSE-scale, Item 2 of the FUF-scale, Item 16 of the FDSscale, and Items 3 and 6 of the FUO-scale. This is also supported when reviewing the standardized factor loadings based upon correlation matrix. With respect to previous translations of the PFAI and its model fits (Correia et al., 2016; Sagar \& Jowett, 2010), the respective items were also reported to show weak factor loadings and excluded from short versions. Hence, model misfit due to the suspected items may rather pertain from the original version of the PFAI than resulting from the German translation.

Further, regarding the quality of single items, Item 16 of the FDS-scale showed the poorest factor loadings, and error variances, analogous to results in previous studies (Conroy \& Metzler, 2003; Sagar \& Jowett, 2010). It was assumed that this may be due to the formulation of Item 16 
Table 9. Hierarchical multiple regression analysis for the prediction of trait anxiety (STAI-T)

\begin{tabular}{|c|c|c|c|c|c|}
\hline Variable & $\beta$ & $R^{2}$ & Adjusted $R^{2}$ & $\Delta R^{2}$ & $\Delta F$ \\
\hline Step 1 & & .01 & .01 & .01 & 1.97 \\
\hline Gender & -.06 & & & & \\
\hline Age & .10 & & & & \\
\hline Step 2 & & .61 & .60 & .59 & $81.17^{\star \star *}$ \\
\hline SCL-27 - DEP & $.34^{\star \star \star}$ & & & & \\
\hline SCL-27 - DYS & $.23^{\star * \star}$ & & & & \\
\hline SCL-27 - VEG & .02 & & & & \\
\hline SCL-27 - AGO & -.03 & & & & \\
\hline SCL-27 - SOC & $.38^{\star \star \star}$ & & & & \\
\hline SCL-27 - MIS & -.01 & & & & \\
\hline Step 3 & & .67 & .66 & .06 & 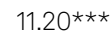 \\
\hline FSE & $.20 * \star \star$ & & & & \\
\hline FDS & .07 & & & & \\
\hline FUF & .05 & & & & \\
\hline FOL & -.02 & & & & \\
\hline FUO & .07 & & & & \\
\hline
\end{tabular}

Note. AGO = agoraphobic symptoms; DEP = symptoms of depression; DYS = dysthymic symptoms; FDS = fear of devaluing one's self-estimate; $F O L=$ fear of important others losing interest; FSE = fear of experiencing shame and embarrassment; FUF = fear of having an uncertain future; FUO = fear of upsetting important others; MIS = symptoms of mistrust; SCL27 = The Symptom-Checklist-27; SOC = sociophobic symptoms; STAI$\mathrm{T}=$ trait-anxiety-scale of the State-Trait Anxiety Inventory; $\mathrm{VEG}=$ vegetative symptoms. ${ }^{*} p<.05,{ }^{* \star} p<.01,{ }^{* \star *} p<.001$

that focuses on the affective experience of hate, rather than on FF threat appraisals (Conroy et al., 2002). Therefore, with respect to the suggestion of Conroy and Metzler (2003), it is recommended to rephrase this item in future application of the PFAI. In line with this recommendation, Item 12 of the FUF-scale, which has been reworded positively in the present study on recommendation of Conroy and Metzler (2003), showed better values regarding its factor loadings, and error variances in relation to the values reported by Sagar and Jowett (2010).
Regarding the construct validity, higher positive correlations between failure appraisals and avoidance goal orientations were expected with respect to previous research (Conroy \& Elliot, 2004; Elliot \& Church, 1997; Elliot \& McGregor, 2001). In the present study, however, correlations with avoidance goals were relatively small and for task-based goals generally negatively correlated. On the other hand, in line with our expectations and previous reports (Conroy \& Elliot, 2004; Elliot \& Church, 1997; Elliot \& McGregor, 2001), the overall PFAI and almost all subscales except for FDS showed significant positive small-sized correlations with SAP goals. Small correlations between $\mathrm{FF}$ and avoidance goals in the present study may be due to sample characteristics. As the current sample included heterogeneous academic backgrounds, various levels of approach and avoidance goal orientations may be represented among participants high in $\mathrm{FF}$, which may have affected the correlations between FF and achievement goal orientations. That is, individuals scoring high in FF do not necessarily choose avoidance goals over approach goals, as this relationship appears to depend on their adopted coping strategies and success orientation. According to the quadripolar model of need achievement, overstrivers tend to choose approach goals, as they are high in FF as well as high in success-orientation, while selfprotectors rather choose avoidance goals, as they are high in FF but low in success-orientation (Covington, 1992; Martin \& Marsh, 2003). Thus, the primary strategy adopted in an academic context may depend on whether one's course of study is highly competitive or not. However, the German version of the AGQ may not have been an appropriate measure to assess the construct validity of the present German version of the PFAI. Therefore, it is suggested to test the inventory against more external criteria regarding achievement goal orientations.

In line with previous research (Conroy, 2001a), overall $\mathrm{FF}$ and all subscales showed significant positive mediumto large-sized correlations with trait anxiety. Beyond that, PFAI subscales, except for FOL, showed unique

Table 10. Overview of model fit values presented in previous validation studies and the present study

\begin{tabular}{|c|c|c|c|c|c|c|}
\hline Country & $\chi^{2 / d f}$ & RMSEA $[90 \% \mathrm{Cl}]$ & SRMR & CFI & GFI & NNFI \\
\hline USA & - & $.05[.04-.06]$ & .06 & .92 & .88 & .91 \\
\hline UK & 2.96 & $.06[.05-.06]$ & .06 & - & - & .89 \\
\hline Turkey* & - & $.09 / .09$ & $.08 / .07$ & $.91 / .96$ & - & $.90 / .95$ \\
\hline Portugal** & - & .04 & - & .96 & .92 & - \\
\hline Romania & 3.05 & $.06[.05-.06]$ & - & .93 & - & - \\
\hline Germany (M3) & 2.84 & .08 [.07-.08] & .07 & .89 & .84 & .88 \\
\hline Germany (M5) & 2.66 & $.07[.07-.08]$ & .08 & .91 & .86 & .89 \\
\hline
\end{tabular}

Note. ${ }^{\star}$ CFA was conducted in two different samples, ${ }^{*}$ adapted 14-item-version of the PFAl. CFI = comparative fit index; $d f=$ degrees of freedom; $\mathrm{GFI}=$ goodness of fit index; NNFI = non-normed fit index; PFAI = Performance Failure Appraisal Inventory; SRMR = standardized root mean square residual; $90 \% \mathrm{Cl}=90 \% \mathrm{Cl}$ of RMSEA; M3 = correlated five-factor model; M5 = bifactor model. 
contributions in the prediction of trait anxiety. Hence, all facets of FF have to be considered separately, reflecting the correlated five-factor structure, when examining the association of FF and anxiety. Regarding the associations of $\mathrm{FF}$ and current psychological symptoms, the largest correlations were observed between $\mathrm{FF}$ and social anxiety symptoms, as well as symptoms of depression and distrust. These results are in line previous research reporting depression, anxiety, low resilience, and interpersonal distress in individuals high in FF (Conroy, 2001b; Conroy et al., 2009; Elliot \& McGregor, 2001; Wright et al., 2009). Again, the subscales of the PFAI contributed differently and uniquely to the prediction of psychopathology. Thus, regarding construct validity, the German version of the PFAI appears to sufficiently measure FF in context of trait anxiety, social anxiety symptoms, symptoms of depression, and symptoms of distrust. Most notably, with respect to our finding of an incremental predictive value, FF may not appear as a single issue and may often be accompanied by other psychological problems, but it still represents a distinct construct that involves cognitive appraisals, emotions, and behavioral and motivational aspects.

The present study has several limitations. The PFAI was validated in a single sample and in a single context, that is, university students in an academic context. Therefore, generalizability of the findings is limited. Moreover, while the sample presented with a wide age-range and various academic backgrounds, it was not representative regarding gender. Hence, results may have been influenced by the large proportion of female participants, as gender differences on subscale-levels have been reported in previous studies regarding FF (Holic, 2018; Kahraman \& Sungur, 2016; Sagar et al., 2011). Also, multivariate analyses revealed gender differences on the PFAI. Unfortunately, however, the current sample was too unbalanced to provide appropriate details and analyses regarding differences in the model structure referring to gender, age, and other factors. Therefore, it is recommended to further examine potential effects of the respective factors in future research. Furthermore, the study relied on participants' self-report that was entirely assessed online. Hence, it is not certain whether participants gave accurate personal information or whether participants fully understood the instructions. Additionally, it cannot be ruled out that some individuals could have participated more than once. Selection factors are also unpredictable in Internet recruitment. Because instructions for the PFAI were adapted to fit into the university context, it remains open to which extent the results of the current study can be generalized to other settings, such as sports or occupation. Future research is needed to examine the impact of instructions on the measure's psychometric properties and its applicability in different contexts. Finally, as there is no instrument (questionnaire or interview) assessing FF in German, the present study could only approximate FF by using scales measuring constructs related to FF to evaluate the construct validity. Hence, further validation against additional external criteria is needed to conclusively evaluate the validity of the PFAI.

In conclusion, the present findings indicate that the German version of the PFAI is a promising measure to assess different aversive consequences that are anticipated upon academic failure among university students. With regards to its field of application, the PFAI shows potential for further expansion, particularly as a prospective diagnostic tool in clinical contexts.

\section{Electronic Supplementary Material}

The electronic supplementary material is available with the online version of the article at https://doi.org/ $10.1027 / 2698-1866 / \mathrm{a} 000018$

ESM 1. German version of the PFAI

ESM 2. PFAI item correlation table

\section{References}

Atkinson, J. W. (1957). Motivational determinants of risk-taking behavior. Psychological Review, 64, Part 1(6), 359-372. https://doi.org/10.1037/h0043445

Birney, R. C., Burdick, H., \& Teevan, R. C. (1969). Fear of failure. Van Nostrand-Reinhold.

Cohen, J. (1988). Statistical power analysis for the behavioral sciences (2nd ed.). Lawrence Erlbaum Associates, Publishers.

Conroy, D. E. (2001a). Progress in the development of a multidimensional measure of fear of failure: The Performance Failure Appraisal Inventory (PFAl). Anxiety, Stress \& Coping, 14(4), 431-452. https://doi.org/10.1080/10615800108248365

Conroy, D. E. (2001b). Fear of failure: An exemplar for social development research in sport. Quest, 53(2), 165-183. https://doi. org/10.1080/00336297.2001.10491736

Conroy, D. E., \& Elliot, A. J. (2004). Fear of failure and achievement goals in sport: Addressing the issue of the chicken and the egg. Anxiety, Stress \& Coping, 17(3), 271-285. https://doi.org/10.1080/ 1061580042000191642

Conroy, D. E., Elliot, A. J., \& Pincus, A. L. (2009). The expression of achievement motives in interpersonal problems. Journal of Personality, 77(2), 495-526.

Conroy, D. E., \& Metzler, J. N. (2003). Temporal stability of performance failure appraisal inventory items. Measurement in Physical Education and Exercise Science, 7(4), 243-261. https:// doi.org/10.1207/S15327841MPEE0704_3

Conroy, D. E., Metzler, J. N., \& Hofer, S. M. (2003). Factorial invariance and latent mean stability of performance failure appraisals. Structural Equation Modeling: A Multidisciplinary Journal, 10(3), 401-422. https://doi.org/10.1207/S15328007SEM1003_4

Conroy, D. E., Willow, J. P., \& Metzler, J. N. (2002). Multidimensional fear of failure measurement: The Performance Failure Appraisal 
Inventory. Journal of Applied Sport Psychology, 14(2), 76-90. https://doi.org/10.1080/10413200252907752

Correia, M., Rosado, A., \& Serpa, S. (2016). Fear of failure in sport: A Portuguese cross-cultural adaptation. Motriz: Revista De Educação Física, 22(4), 376-382. https://doi.org/10.1590/S19806574201600040024

Covington, M. V. (1992). Making the grade: A self-worth perspective on motivation and school reform. Cambridge University Press.

Cucina, J., \& Byle, K. (2017). The bifactor model fits better than the higher-order model in more than $90 \%$ of comparisons for mental abilities test batteries. Journal of Intelligence, 5(3), 27. https:// doi.org/10.3390/jintelligence5030027

De Castella, K., Byrne, D., \& Covington, M. (2013). Unmotivated or motivated to fail? A cross-cultural study of achievement motivation, fear of failure, and student disengagement. Journal of Educational Psychology, 105(3), 861-880. https://doi.org/10. 1037/a0032464

Elliot, A. J., \& Church, M. A. (1997). A hierarchical model of approach and avoidance achievement motivation. Journal of Personality and Social Psychology, 72(1), 218-232. https://doi.org/10.1037/ 0022-3514.72.1.218

Elliot, A. J., \& McGregor, H. A. (2001). A $2 \times 2$ achievement goal framework. Journal of Personality and Social Psychology, 80(3), 501-519. https://doi.org/10.1037/0022-3514.80.3.501

Elliot, A. J., Murayama, K., \& Pekrun, R. (2011). A $3 \times 2$ achievement goal model. Journal of Educational Psychology, 103(3), 632-648. https://doi.org/10.1037/a0023952

Elliot, A. J., \& Thrash, T. M. (2004). The intergenerational transmission of fear of failure. Personality and Social Psychology Bulletin, 30(8), 957-971. https://doi.org/10.1177/0146167203262024

Flora, D. B. (2020). Your coefficient alpha is probably wrong, but which coefficient omega is right? A tutorial on using $\mathrm{R}$ to obtain better reliability estimates. Advances in Methods and Practices in Psychological Science, 3(4), 484-501. https://doi.org/10.1177/ 2515245920951747

Gelbort, K. R., \& Winer, J. L. (1985). Fear of success and fear of failure: A multitrait-multimethod validation study. Journal of Personality and Social Psychology, 48(4), 1009-1014. https://doi. org/10.1037/0022-3514.48.4.1009

Greiff, S., \& Heene, M. (2017). Why psychological assessment needs to start worrying about model fit. European Journal of Psychological Assessment, 33(5), 313-317. https://doi.org/10.1027/ 1015-5759/a000450

Haghbin, M., McCaffrey, A., \& Pychyl, T. A. (2012). The complexity of the relation between fear of failure and procrastination. Journal of Rational-Emotive \& Cognitive-Behavior Therapy, 30(4), 249-263. https://doi.org/10.1007/s10942-012-0153-9

Hardt, J., Egle, U. T., Kappis, B., Hessel, A., \& Brähler, E. (2004). Die Symptom-Checkliste SCL-27 - Ergebnisse einer deutschen Repräsentativbefragung [Symptom Checklist SCL-27: Results of a representative German survey]. Psychotherapie, Psychosomatik, Medizinische Psychologie, 54(5), 214-223. https://doi.org/ 10.1055/s-2003-814786

Harrer, K. (2012). Empirische Überprüfung des $3 \times 2$ Modells der Zielorientierungen und dessen Zusammenhang mit Lernfreude und Langeweile [Empirical review of the $3 \times 2$ model of goal orientation and its connection with the joy of learning and boredom] [Thesis]. University of Vienna. http://othes.univie.ac. at/24423/1/2012-12-16_0606411.pdf

Heene, M., Hilbert, S., Draxler, C., Ziegler, M., \& Bühner, M. (2011). Masking misfit in confirmatory factor analysis by increasing unique variances: A cautionary note on the usefulness of cutoff values of fit indices. Psychological Methods, 16(3), 319-336. https://doi.org/10.1037/a0024917

Holic, R. (2018). The validation of performance failure appraisal inventory for the educational context of high school students in
Romania. Studia Universitatis Babeş-Bolyai Psychologia-Paedagogia, 63(1), 49-78. https://doi.org/10.24193/subbpsyped.2018.1.03

Hooper, D., Coughlan, J., \& Mullen, M. R. (2008). Structural equation modelling: Guidelines for determining model fit. The Electronic Journal of Business Research Methods, 6(1), 53-60.

Hu, L. t., \& Bentler, P. M. (1999). Cutoff criteria for fit indexes in covariance structure analysis: Conventional criteria versus new alternatives. Structural Equation Modeling: A Multidisciplinary Journal, 6(1), 1-55. https://doi.org/10.1080/10705519909540118

IBM Corporation. (2012). IBM SPSS statistics for Windows (Version 21.0).

Kahraman, N., \& Sungur, S. (2016). Adaptation of the Performance Failure Appraisal Inventory (PFAl) into Turkish. Journal of Kirsehir Education Faculty, 17(3), 223-239.

Laux, L., Glanzmann, P., Schaffner, P., \& Spielberger, C. D. (1981). Das State-Trait-Angstinventar (STAI) [The State-Trait Fear Inventory]. Hogrefe.

Martin, A. J., \& Marsh, H. W. (2003). Fear of failure: Friend or foe? Australian Psychologist, 38(1), 31-38. https://doi.org/10.1080/ 00050060310001706997

McGregor, H. A., \& Elliot, A. J. (2005). The shame of failure: Examining the link between fear of failure and shame. Personality and Social Psychology Bulletin, 31(2), 218-231. https://doi.org/10. $1177 / 0146167204271420$

Moshagen, M., \& Auerswald, M. (2018). On congruence and incongruence of measures of fit in structural equation modeling. Psychological Methods, 23(2), 318-336. https://doi.org/10.1037/met0000122

Muthén, B. O. (1989). Latent variable modeling in heterogeneous populations. Psychometrika, 54(4), 557-585. https://doi.org/10. 1007/BF02296397

RStudio Team. (2020). RStudio: Integrated development environment for $R$ (Version 3.4.3).

Sagar, S. S., Boardley, I. D., \& Kavussanu, M. (2011). Fear of failure and student athletes' interpersonal antisocial behaviour in education and sport. The British Journal of Educational Psychology, 81(pt 3), 391-408. https://doi.org/10.1348/2044-8279.002001

Sagar, S. S., \& Jowett, S. (2010). Validation of a multidimensional measure of fear of failure in a British sample: The Performance Failure Appraisal Inventory (PFAI). International Journal of Coaching Science, 4(1), 49-63.

Sagar, S. S., \& Stoeber, J. (2009). Perfectionism, fear of failure, and affective responses to success and failure: The central role of fear of experiencing shame and embarrassment. Journal of Sport \& Exercise Psychology, 31(5), 602-627. https://doi.org/10. 1123/jsep.31.5.602

Wagenmakers, E.-J., \& Farrell, S. (2004). Aic model selection using Akaike weights. Psychonomic Bulletin \& Review, 11(1), 192-196. https://doi.org/10.3758/BF03206482

Wright, A. G. C., Pincus, A. L., Conroy, D. E., \& Elliot, A. J. (2009). The pathoplastic relationship between interpersonal problems and fear of failure. Journal of Personality, 77(4), 997-1024. https:// doi.org/10.1111/j.1467-6494.2009.00572.x

\section{History}

Received May 4, 2021

Revision received August 14, 2021

Accepted September 28, 2021

Published online December 16, 2021

\section{Publication Ethics}

Each participant read and signed an informed consent form that was approved by the Ethics Committee of Bielefeld University (protocol number: EUB 2020-027).

\section{Funding}

We acknowledge support for the publication costs by the Open Access Publication Fund of Bielefeld University. 


\section{ORCID}

Christine Henschel

(iD) https://orcid.org/0000-0002-2850-6850

Benjamin Iffland

(D) https://orcid.org/0000-0003-3122-005X

\section{Christine Henschel}

Department of Psychology

Bielefeld University

Postbox 100131

33501 Bielefeld

Germany

christine.henschel@uni-bielefeld.de 